Somnambulism (sleepwalking) is a disorder of arousal that falls under "parasomnia" group and is more common in children. These phenomena occur as primary sleep events or secondary to systemic disease or can be drug induced. Medications that can cause sleepwalking include neuroleptics, hypnotics, lithium, amitriptyline, and $\beta$-blockers. 1 This report presents an unusual adverse effect of topiramate on sleep in a patient with migraine. Keywords: Topiramate, somnambulism, migraine, idiosyncratic reaction, adverse effect

Citation: Mathew T; Sarma GRK; Nadig R; Varghese R. Topiramate-induced somnambulism in a migraineur: a probable idiosyncratic adverse effect. J Clin Sleep Med 2012;8(2):197-198.
Nomnambulism (sleepwalking) is a disorder of arousal that $\checkmark$ falls under "parasomnia" group and is more common in children. These phenomena occur as primary sleep events or secondary to systemic disease or can be drug induced. Medications that can cause sleepwalking include neuroleptics, hypnotics, lithium, amitriptyline, and $\beta$-blockers. ${ }^{1}$ This report presents an unusual adverse effect of topiramate on sleep in a patient with migraine.

\section{REPORT OF CASE}

A 35-year-old woman suffering from migraine without aura for past 7 years was started on topiramate $25 \mathrm{mg}$ daily for prophylaxis. The patient started the drug and had an uneventful sleep on the first day. The second day she took topiramate 25 $\mathrm{mg}$ at 22:00 and went to bed. About $2 \mathrm{~h}$ after sleep, her husband noticed that she was not in the bed and found her walking around in the room. She was not responding to his call and continued to walk in a "trance-like" state for about half an hour. Her husband forced her back to bed and she continued to sleep. Next day morning she was totally unaware of what had happened in the night. She consumed topiramate $25 \mathrm{mg}$ on the third day and had similar episode on that night. The duration and time of the event were same as in the first night, as reported by the patient's husband. She was apprehensive about continuing topiramate and stopped it on her own on the fourth day. She had no further episodes. Her habitual sleep duration was about 6-7 hours. Patient did not report any change in the sleep duration after initiation of topiramate. She did not report any change in her stress levels during the days she was taking topiramate. There was no history suggestive of any sleep related breathing disorder or somniloquy. She did not report any flu-like symptoms after initiation of topiramate. She denied any prior history of somnambulism or family history of sleep disorders. She was not taking any other medications. A challenge with topiramate with polysomnography was suggested, but the patient was unwilling to take the medication again.

\section{DISCUSSION}

This 35-year-old woman with migraine is the second case of topiramate-induced somnambulism reported in literature. Interestingly the previous case report is also from India, involving a 27 -year-old man with chronic migraine. ${ }^{2}$ The fact that sleepwalking occurred on the second day with a small dose of topiramate $(25 \mathrm{mg})$ suggests an idiosyncratic reaction rather than a dose-dependent response. Frontal lobe epilepsy is an important differential diagnosis for somnambulistic episodes. But it is very rare for frontal lobe epilepsies to present only with pure somnambulism, and they are of briefer duration. There was no history of seizures in the past or family. There was no evidence of tonic clonic movements, automatisms, or urinary incontinence. Topiramate is also an antiepileptic, and to induce seizure at such a low dose is very unlikely.

Topiramate is a drug commonly prescribed for migraine prophylaxis and epilepsy by physicians and neurologists. Topiramate has also been reported to be useful for treatment of nocturnal eating syndrome and sleep-related eating disorder (SRED). ${ }^{3}$ Topiramate has multiple mechanisms of action, including voltage-sensitive sodium channel blockade, calcium channel inhibition, increase in potassium conductance, GABA mediated chloride current increment, glutamate-mediated neurotransmission inhibition, and carbonic anhydrase isoenzyme inhibition. Topiramate increases the GABA-mediated chloride flux in cultured mouse neurons. It exerts a negative modulatory effect on excitatory glutamate neurotransmission. The inhibitory effect is mediated by the kainate AMPA subtype of glutamate receptor.

The exact mechanism of topiramate induced somnambulism is not known. The initiation of NREM sleep is gradual and is characterized by slowing of the brain waves on EEG. Very high amplitude delta waves occur in stages 3 and 4 of NREM. Somnambulism typically occurs during the first 3 hours of sleep, when stages 3 and 4 of NREM are most prevalent. The effects of topiramate on sleep are practically unknown. Most of the 
patients who undergo polysomnography are refractory seizure patients on multiple antiepileptic drugs. So the individual effect of antiepileptic drugs on sleep are difficult to ascertain. ${ }^{4}$ Riluzole, a glutamate antagonist, is known to enhance the duration of slow wave sleep in rats. ${ }^{5}$ Topiramate by its anti-glutaminergic effect may also increase the duration of slow wave sleep.

Migraine and sleepwalking have an interesting relation. Serotonin which plays an important role in migraine pathogenesis, is also involved in slow wave sleep. There is a six-fold higher prevalence of somnambulism in patients with migraine. The predisposing factors and physiological pathways of migraine and somnambulism appears similar. ${ }^{6}$ The cerebral serotonergic system may play an important role in the pathophysiology of sleepwalking. Cape and Jones revealed that microinjection of serotonin during sleep in cholinergic basalis neurons increases EEG delta activity in rats. Moreover, in one-half of rats injected with serotonin, these authors observed anomalous wake episodes associated with high delta activity. These wake episodes were characterized by open eyes and quiet behavior and in fact resembled sleepwalking in humans. ${ }^{7}$

Serotonin, GABA, and glutamate play an important role in wakefulness and sleep. Serotonin neurons are most active during wakefulness and fire most rapidly during continuous motor activity such as walking or running. GABA has a significant role in NREM sleep while glutamate plays a pivotal role in REM sleep. During NREM sleep, GABA has been hypothesized to play key roles in the deactivation of wake-related arousal systems and in the generation of intrinsic thalamocortical oscillations. The excitatory amino acid glutamate widely interacts with cholinergic and cholinoceptive neurons to generate the exponential increase of mesopontine and pontine reticular activity associated with REM sleep activation. Glutamatergic cells of the pons transmit signals to inhibitory glycinergic and GABAergic cells of the medulla, which in turn, suppress somatic motor neurons to produce REM sleep atonia. Topiramate, a pro-GABA and anti-glutamate agent, is also known to increase serotonin levels in children with migraine. ${ }^{8}$ We hypothesize that topiramate by its pro-GABA and anti-glutamate effect promotes NREM sleep, and by increasing serotonin levels favors motor activity. This may result in the intrusion of motor activity during NREM sleep, resulting in sleepwalking.

The phenomenon of drugs used for treating a particular disorder precipitating the same disorder is not rare in medical literature. Zolpidem, though used in the treatment of insomnia, is also known to precipitate somnambulism. Medications used to treat migraine such as propranolol and amitriptyline have sometimes induced, but most often cured, sleepwalking or other parasomnias. ${ }^{6}$ Topiramate, though reported to be efficient in treating SRED, now is reported to produce somnambulism. This may due to the differences in the etiopathogenesis of sleepwalking and SRED at the receptor and neurotransmitter level. Dopaminergic/serotonergic mechanisms may be more important in SRED, while GABAergic/glutaminergic mechanisms may be more important in somnambulism.

This adverse effect of topiramate on sleep needs further study, as it is a useful drug in the field of epilepsy, headache, and sleep. It will be worthwhile to counsel the patients, especially those with chronic migraine, about this rare side effect because of the potential medicolegal implications.

\section{REFERENCES}

1. Pressman MR. Factors that predispose prime and precipitate NREM parasomnias in adults: clinical and forensic implications. Sleep Med Rev 2007;11:5-30.

2. Varkey BM, Varkey LM. Topiramate induced somnambulism and automatic behaviour. Indian J Med Sci 2003;57:508.

3. Winkelman JW. Treatment of nocturnal eating syndrome and sleep-related eating disorder with topiramate. Sleep Med 2003;4:243-6.

4. Neufeld MY, Kogan E, Chistik V, Korezyn AD. Comparison of effects of vigabatrin, lamotrigine and topiramate on quantitative EEGs in patients with epilepsy. Clin Neuropharmacol 1999;22:80-6.

5. Stutzmann JM, Lucas M, Blanchard JC, Laduron PM. Riluzole, a glutamate antagonist, enhances slow wave sleep and REM sleep in rats. Neurosci Lett 1988:88:195-200.

6. Casez $\mathrm{O}$, Danachet $\mathrm{Y}$, Besson G. Migraine and somnambulism. Neurology 2005;65:1334-5

7. Cape EG, Jones BE. Differential modulation of high frequency gamma electroencephalogram activity and sleep-wake state by noradrenaline and serotonin microinjections into the region of cholinergic basalis neurons. $J$ Neurosci 1998;18:2653-66

8. Grosso S, Blardi P, Battaglini M, et al. Topiramate effects on plasma serotonin levels in children with epilepsy. Epilepsy Res 2008;81:148-54.

\section{ACKNOWLEDGMENTS}

Work for this study was performed at St. John's Medical College Hospital.

\section{SUBMISSION \& CORRESPONDENCE INFORMATION}

\section{Submitted for publication July, 2011}

Submitted in final revised form October, 2011

Accepted for publication November, 2011

Address correspondence to: Dr. Thomas Mathew, Associate Professor, Department of Neurology, St. John's Medical College Hospital, Sarjapur Road, Bangalore-560034; Te,: +91 80 22065330; Fax: +91 80 25634479; E-mail: chakkuthom@hotmail.com

\section{DISCLOSURE STATEMENT}

This was not an industry supported study. The authors have indicated no financial conflicts of interest. 